Компьютерная томография в диагностике и лечении воспалительных осложнений дивертикулярной болезни ободочной кишки

\author{
Карпухин О.Ю. ${ }^{1,2}$, Юсупова А.Ф.',2, Панкратова Ю.С. ${ }^{1}$, Черкашина М.И. ${ }^{2}$, \\ Ахмадуллина А.А.' \\ 'ФГБОУ ВО Казанский ГМУ Минздрава России (ул. Бутлерова, д. 49, г. Казань, 420012, Республика \\ Татарстан, Россия) \\ ${ }^{2}$ ГАУЗ «Республиканская клиническая больница» МЗ РТ (Оренбургский тракт, д. 138, г. Казань, 420064, \\ Республика Татарстан, Россия)
}

PEЗЮМЕ ЦЕЛЬ: оценить роль компьютерной томографии в лечебно-диагностическом алгоритме при осложненных формах дивертикулярной болезни ободочной кишки (ДБОК).

ПАЦИЕНТЫ И МЕТОДЫ: в Исследование включены 165 пациентов с осложнениями ДБОК, находившиеся на стационарном исследовании в 2014-2020 г2. В плановом порядке были госпитализированы 15 (9,1\%) пациентов, по экстренным показаниям - 150 (90,9\%). Показаниями к госпитализации послужили воспалительные осложнения ДБОК. Рентгеновская компьютерная томография с внутривенным контрастированием выполнена 89 (53,9\%) пациентам. Исследование выполняли на 64-срезовом компьютерном томографе «Philips Brilliance 64» с использованием внутривенного болюсного введения неионного йодосодержащего контрастного препарата. Отсутствие РКТ у остальных пациентов обусловлено наличием классической симптоматики обострения дивертикулита с ранее верифицированным диагнозом ДБОК, наличием информативного заключения УзИ, а также отказом пациентов от РКТ.

РЕЗУЛЬТАТЫ: проведенная РКТ диагностика позволила верифицировать наличие дивертикулов у пациентов, выявить характерные РКТ признаки и патогномоничные симптомы воспалительных осложнений ДБОК, а также установить тяжесть возникших осложнений. Описаны характерные признаки деструкции дивертикула и развивающихся при этом осложнений - инфильтрата, абсцесса, перитонита, свища. Помимо диагностики, РКТ позволяет определять тактику лечения, уточняя показания к оперативному вмешательству. Кроме того, некоторые осложнения ДБОК, выявленные в ходе РКТ, можно рассматривать в качестве предиктора неэффективности консервативного лечения, что требует активных действий со стороны хирурга.

ЗАКЛЮЧЕНИЕ: РКТ — эффективный метод диагностики ДБОК, позволяющий своевременно установить клинический вариант воспалительного осложнения, определить показания к оперативному лечению и прогнозировать вероятность рецидива.

кЛЮчЕВЫЕ СЛОвА: осложненные формы дивертикулярной болезни ободочной кишки, РКТ-диагностика, предикторы рецидива КОНФЛИКТ ИНТЕРЕСОВ: Авторы заявляют об отсутствии конфликта интересов.

ИССЛЕДОВАНИЕ НЕ ИМЕЛО ИСТОЧНИКА ФИНАНСИРОВАНИЯ.

для цитИРОВАния: Карпухин 0.Ю., Юсупова А.Ф., Панкратова Ю.С., Черкашина М.И., Ахмадуллина А.А. Компьютерная томография в диагностике и лечении воспалительных осложнений дивертикулярной болезни ободочной кишки. Колопроктология. 2021; т. 20, № 4, c. 34-41. https://doi.org/10.33878/2073-7556-2021-20-4-34-41

\title{
Computer tomography in diagnostics and treatment of inflammatory complications of diverticular disease of the colon
}

\section{Oleg Yu. Karpukhin 1,2, Alsu F. Yusupova 1,2, Yulia S. Pankratova', Malika I. Cherkashina ${ }^{2}$, Albina A. Akhmadullina'}

'Kazan State Medical University (Butlerov Street, 49, Kazan, 420012, Russia)

${ }^{2}$ Republican Clinical Hospital (Orenburg tract, 138, 420064, Kazan, Russia)

ABSTRACT AIM: to evaluate the role of computed tomography (CT) in the treatment and diagnostic algorithm in patients with complicated diverticular disease (CDD).

PATIENTS AND METHODS: during the period from 2014 to 2020, 165 hospitalized patients with complications of 
CDD included in the study. Fifteen (9.1\%) patients were hospitalized for elective indications and 150 (90.9\%) as emergencies. The indications for hospitalization were inflammatory complications of CDD. Computed tomography with intravenous contrast was performed in 89 (53.9\%) patients. The study was performed on a 64-slice CT "Philips Brilliance 64" with intravenous bolus injection of a low-osmolar iodine-containing contrast agent. The absence of the $C T$ in the remaining patients is due to the presence of classical symptoms of acute diverticulitis with a previously verified diagnosis of CDD, the presence of an informative transabdominal ultrasound, as well as the refusal of patients from CT.

RESULTS: the CT allowed to verify the presence of diverticula in the patients, to reveal the distinctive CT signs and pathognomonic symptoms of inflammatory complications of CDD, as well as to establish the severity of the complications that occurred. The specific signs of the destruction of the diverticulum and the complications developed were abdominal mass, abscess, peritonitis, and fistula. Besides the diagnostic value, CT scan permitted to choose the treatment approach and to clarify indications for surgery. Besides that, some CDD complications revealed by CT were considered as a predictor of ineffectiveness of conservative treatment, which requires surgery.

CONCLUSION: CT is a valuable diagnostic method for CDD which allows to determine timely the clinical form of inflammatory complication, to find out indications for surgery and to predict high risk of recurrence.

KEYWORDS: complicated forms of colonic diverticular disease, CT-diagnostics, predictors of recurrence

CONFLICT OF INTEREST: The authors declare no conflict of interest

THE STUDY HAD NO SOURCE OF FUNDING.

FOR CITATION: Karpukhin 0.Yu., Yusupova A.F., Pankratova Yu.S., Cherkashina M.I., Akhmadullina A.A. Computer tomography in diagnostics and treatment of inflammatory complications of diverticular disease of the colon. Koloproktologia. 2021;20(4):34-41. (in Russ.). https://doi.org/10.33878/2073-7556-2021-20-4-34-41

АДРЕС ДЛЯ ПЕРЕПИСКИ: Карпухин Олег Юрьевич, ФГБОУ ВО Казанский ГмУ Минздрава России ,ул. Бутлерова, д. 49, Казань, 420012, Республика Татарстан, Россия; e-mail: oleg_karpukhin@mail.ru

ADDRESS FOR CORRESPONDENCE: Karpukhin Oleg Yurevich, Kazan State Medical University, Butlerov Street, 49, Kazan, 420012, Russia; e-mail: oleg_karpukhin@mail.ru

Дата поступления - 31.05.2021

Received - 31.05.2021
После доработки - 02.08.2021

Revised - 02.08.2021

\author{
Принято к публикации - 30.11.2021
}

Accepted for publication - 30.11.2021

\section{ВВЕДЕНИЕ}

Дивертикулярная болезнь ободочной кишки (ДБОК) - одно из самых распространенных заболеваний в западной цивилизации. Его отличает многообразие проявлений от бессимптомного течения заболевания до осложненных форм, требующих неотложных хирургических вмешательств. Так, до 20\% пациентов с дивертикулярной болезнью страдают от развития острого дивертикулита, который в результате воспалительной деструкции дивертикула может осложниться перитонитом, формированием абсцесса или патологического соустья [1]. Осложненные формы ДБОК становятся частой причиной госпитализации пациентов по экстренным показаниям в отделения ургентной хирургии или колопроктологии, где проводится сложное, нередко многоэтапное хирургическое лечение. Таким образом, широкая распространенность заболевания, частое развитие тяжелых осложнений, требующих неотложных оперативных вмешательств с длительной послеоперационной реабилитацией пациентов, обусловливают необычайную актуальность ранней диагностики и своевременного лечения пациентов с осложнениями ДБОК.

В настоящее время за рубежом рентгеновская компьютерная томография (РКТ) брюшной полости и малого таза с внутривенным контрастированием рассматривается в качестве эффективного метода визуальной оценки дивертикулярного воспаления, позволяющего не только верифицировать наличие острого дивертикулита, но и определить клинический вариант воспалительного осложнения, проводить дифференциальную диагностику, а при наличии таких осложнений как перфорация и абсцесс, влиять на выбор лечебной тактики [2-4]. При этом чувствительность РКТ при дивертикулярном воспалении варьируется от 79\% до 99\% [3,5].

В отечественной литературе вопрос проведения РКТ при наличии клинической картины острого дивертикулита остается дискутабельным [6]. Не решен вопрос, насколько целесообразно выполнение РКТ органов брюшной полости для уточнения диагноза при первичном обращении пациента. Сообщается о предикторах, с высокой вероятностью указывающих на острый дивертикулит, таких как боли в левой подвздошной области, усиливающиеся при движении, возраст пациента старше 50 лет, эпизоды острого дивертикулита в анамнезе, болезненность при пальпации в нижних отделах живота, повышенный уровень С-реактивного белка (выше 50 мг/л), отсутствие рвоты [7]. Авторы исследования заявляют, что при наличии в клинической картине этих признаков дополнительная 
визуализация может быть исключена. В других исследованиях, напротив, утверждается, что точность диагноза острого дивертикулита на основании лишь клинической оценки низкая, с чувствительностью 64\% и $68 \%[8,9]$. Поэтому большинство авторов при диагностике острых воспалительных осложнений ДБОК отдают предпочтение РКТ [5,10,11], что обусловлено возможностью метода не только обнаруживать дивертикулы, но и с высокой степенью достоверности определять вариант воспалительных осложнений ДБОК, дифференцировать дивертикулярную болезнь с другими заболеваниями кишечника [12].

\section{ЦЕЛЬ ИССЛЕДОВАНИЯ}

Оценить роль компьютерной томографии в лечебнодиагностическом алгоритме при осложненных формах дивертикулярной болезни ободочной кишки.

\section{ПАЦИЕНТЫ И МЕТОДЫ}

С 2014 по 2020 гг. на стационарном лечении в отделении колопроктологии ГАУЗ РКБ МЗ РТ находились 165 пациентов с ДБОК. При этом 7 (4,2\%) из них госпитализировались по этому поводу неоднократно. Мужчин было 55 (33,3\%), женщин - 110 (66,7\%). Возраст пациентов колебался от 26 до 91 лет, средний возраст составил 60,8 $\pm 11,1$ лет.

В плановом порядке госпитализировано 15 (9,1\%) пациентов, по экстренным показаниям - 150 (90,9\%). Причиной госпитализации больных в отделение хирургического профиля были: острый дивертикулит 78 (47,3\%) (58 - первичный приступ, 20 - обострение хронического дивертикулита); инфильтрат брюшной полости - 31 (18,8\%); толстокишечное кровотечение - 28 (17\%); абсцесс брюшной полости или брыжейки - 15 (9,1\%); перфорация дивертикула, осложненная перитонитом - 6 (3,6\%); толстокишечно-мочепузырный свищ - 4 (2,4\%); стриктура сигмовидной кишки, осложненная хронической - $2(1,2 \%)$ либо острой кишечной непроходимостью - 1 (0,6\%). у 7 пациентов с ДБОК, поступавших в клинику, неоднократно причиной госпитализаций были: у одного пациента - рецидивирующее толстокишечное кровотечение (стационарное лечение проводилось 4 раза), у 6 пациентов - обострение хронического дивертикулита (при этом у одного пациента стационарное лечение проводилось трижды, а у 5 - дважды).

РКТ с внутривенным контрастированием выполнена у 89 (53,9\%) больных при первичной госпитализации в отделение колопроктологии РКБ МЗ РТ. У оставшейся группы пациентов КТ исследование не проводилось, что было обусловлено наличием классической симптоматики обострения дивертикулита с ранее верифицированным диагнозом ДБОК, а также наличием информативного, не вызывающего сомнения заключения трансабдоминального УЗИ. Двое больных категорически отказались от РКТ в связи с боязнью лучевой нагрузки. В группе пациентов с толстокишечным кровотечением методом первичной диагностики была колоноскопия.

Исследование выполняли на 64-срезовом компьютерном томографе «Philips Brilliance 64» с использованием внутривенного болюсного введения неионного низкоосмолярного йодсодержащего контрастного препарата (йопромид, йоверсол, йогексол). При подозрении на острую хирургическую ситуацию, развившуюся в результате ДБОК, РКТ выполняли непосредственно при поступлении пациента в клинику. За 30 минут до исследования пациент выпивал 500 мл воды с водорастворимым рентгеноконтрастным препаратом. При плановых исследованиях КТ проводилась натощак, без подготовки кишечника. Накануне исследования пациент выпивал 250 мл воды с водорастворимым йодосодержащим контрастным препаратом и еще 250 мл - за 30 минут до исследования. При подозрении на наличие внутреннего свища дивертикулярного происхождения в прямую кишку ретроградно вводили 150 мл слабого раствора водорастворимого рентгеноконтрастного вещества.

\section{РЕЗУЛЬТАТЫ И ОБСУЖДЕНИЕ}

Распределение пациентов, у которых в диагностике ДБОК использовали РКТ, по группам с учетом варианта воспалительных осложнений и проведенного лечения отражено в таблице 1. У одного пациента с клиникой обострения хронического рецидивирующего дивертикулита РКТ не выявила признаков дивертикулов, диагноз верифицирован при ирригографии и интраоперационно. РКТ у пациентов с различными вариантами осложнений ДБОК позволила выделить характерные КТ признаки острого дивертикулита, дивертикулярной деструкции, инфильтрата, абсцесса, стриктуры и свища дивертикулярного происхождения.

При остром дивертикулите на компьютерных томограммах по окружности кишечной стенки визуализировались разнокалиберные мешотчатые выпячивания с нечеткими контурами, а также утолщение стенок пораженного сегмента ободочной кишки с сужением её просвета (Рис. 1). Утолщение стенок ободочной кишки, достигавшее 4 мм и более, выявлено у 82 (92,1\%) пациентов, при этом протяженность воспалительного сегмента ободочной кишки варьировалась от 20 до 56 мм. Согласно данным Kandagatla P.G., Stefanou A.G. (2018), протяженность 
Таблица 1. Воспалительные осложнения ДБОК и виды лечения в группе пациентов с РКТ брющной полости и полости малого таза

Table 1. CDD and types of treatment in the group of patients with abdominal and pelvic CT scan

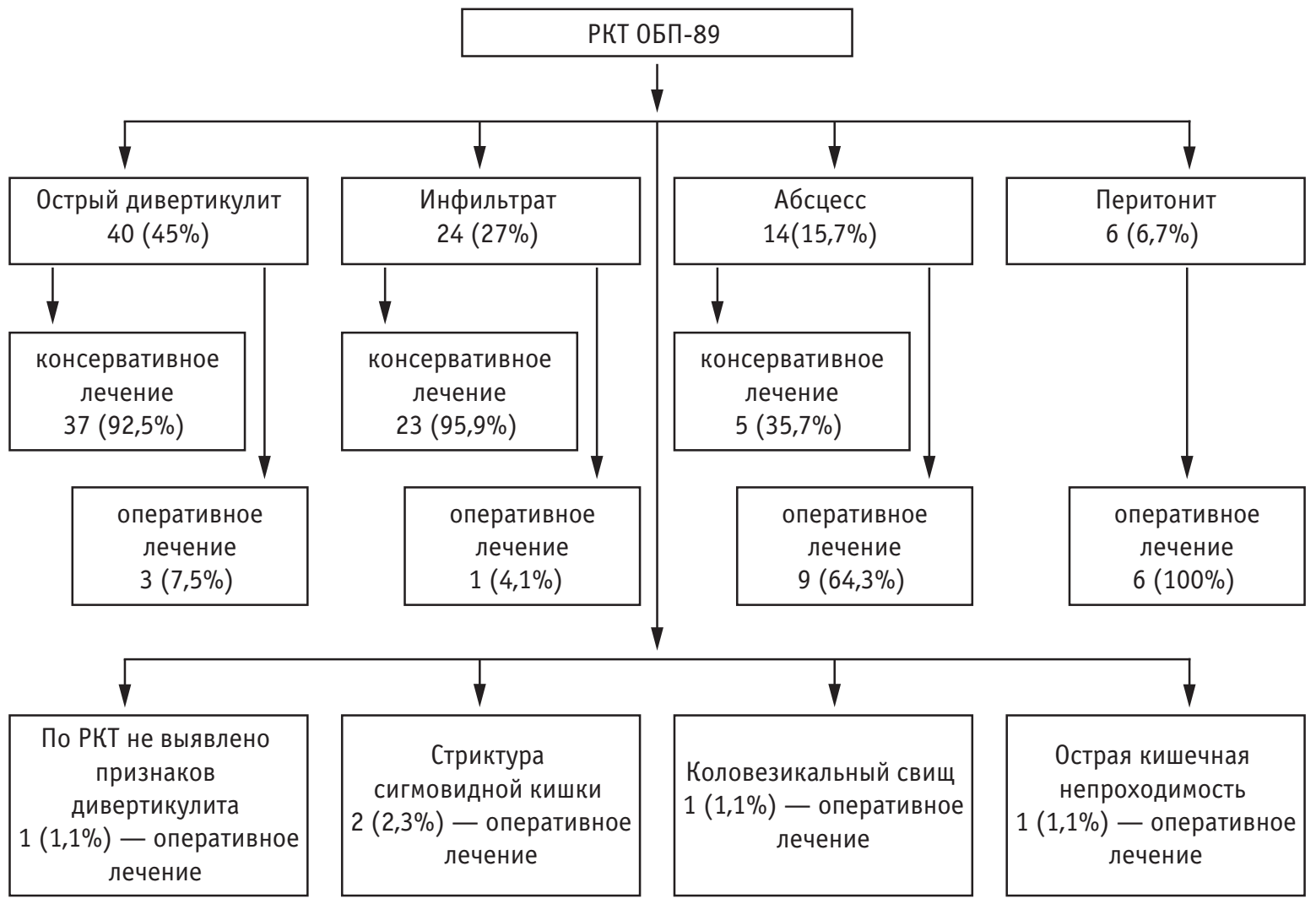

воспалительного поражения кишечной стенки более 5 см при ДБОК увеличивает вероятность возникновения рецидива заболевания [13].

Признаками распространения воспаления за пределы дивертикула с формированием инфильтрата на КТ являются воспалительные изменения параколической клетчатки, проявляющиеся неравномерностью, тяжистостью её структуры за счет множества прослоек жидкости на фоне отека жировой ткани в результате воспалительных изменений дивертикулов, так называемый «грязный жир» [2,5] (Рис. 2).

Грозным осложнением ДБОК является перфорация дивертикула с развитием воспалительных изменений в брюшной полости или в брыжейке ободочной кишки (в зависимости от расположения дивертикула в стенке кишки по отношению к tenia antimesenteric или tenia mesenteric). Признаками деструкции дивертикула по данным КТ в наших наблюдениях были инфильтрация параколической клетчатки с включением пузырьков газа или скоплением контрастного вещества вне просвета кишки. При деструкции дивертикула, расположенного по брыжеечному краю кишки, поступление кишечной микрофлоры и газа чаще происходит в жировые пространства брыжейки ободочной кишки. При этом клинические проявления возникшего

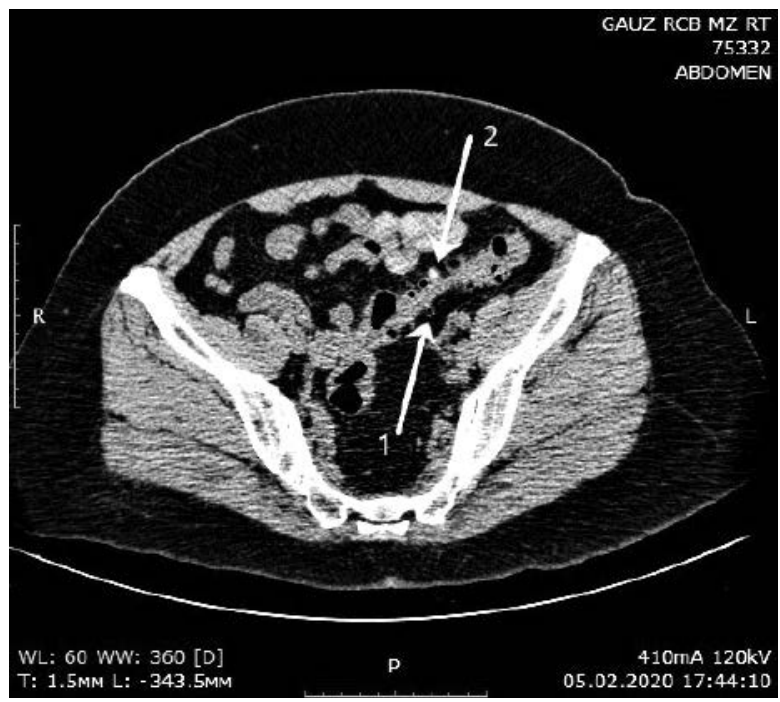

Рисунок 1. Томограмма пациентки Ж, 53 лет. Аксиальный срез. Дивертикулярная болезнь левой половины ободочной кишки, осложнённая дивертикулитом. Множественные дивертикулы с утолщенными стенками, заполненные воздухом (стрелка 1) и копролитами (стрелка 2)

Figure 1. CT of patient J, 53 years old. Axial section. Diverticular disease of the left colon complicated by diverticulitis. Multiple diverticula with thickened walls filled with air (arrow 1) and coprolites (arrow 2). 


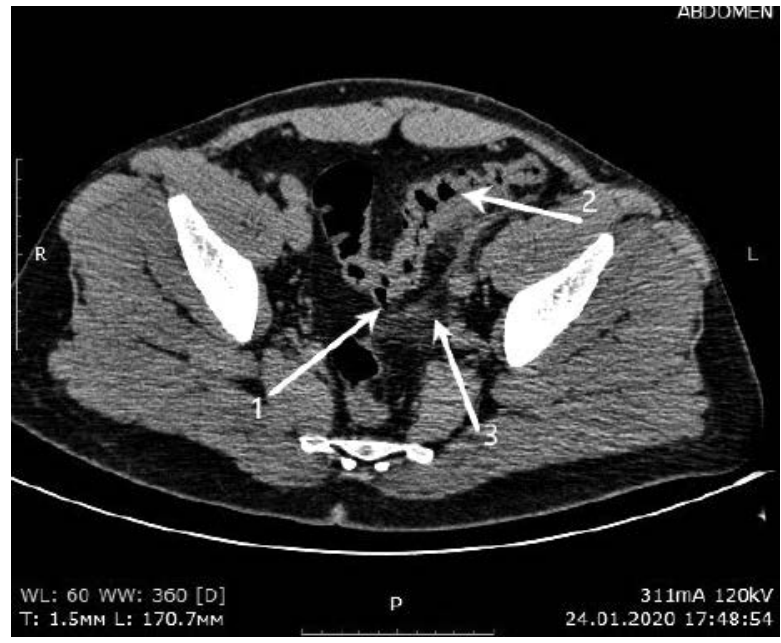

Рисунок 2. Томограмма пациента К., 42 лет. Аксиальный срез. Дивертикулярная болезнь сигмовидной кишки, осложненная дивертикулитом. Дивертикул сигмовидной кишки (стрелка 1), утолщение стенок сигмовидной кишки, приводящее к сужению её просвета (стрелка 2), инфильтрация клетчатки (стрелка 3)

Figure 2. CT of patient K., 42 years old. Diverticular disease of the sigmoid colon complicated by diverticulitis. Diverticulum of the sigmoid colon (arrow 1), significant thickening of the walls of the sigmoid colon, narrowing of its lumen (arrow 2), infiltration of pericolic fat (arrow 3).

осложнения могут быть лишены классических признаков местного, тем более генерализованного перитонита. На КТ можно наблюдать отечность корня брыжейки ободочной кишки, а также так называемый симптом «запятой» $[2,14]$ - утолщение и скопление жидкости в области фасции левого латерального канала или позади ободочной фасции (Рис. 3).

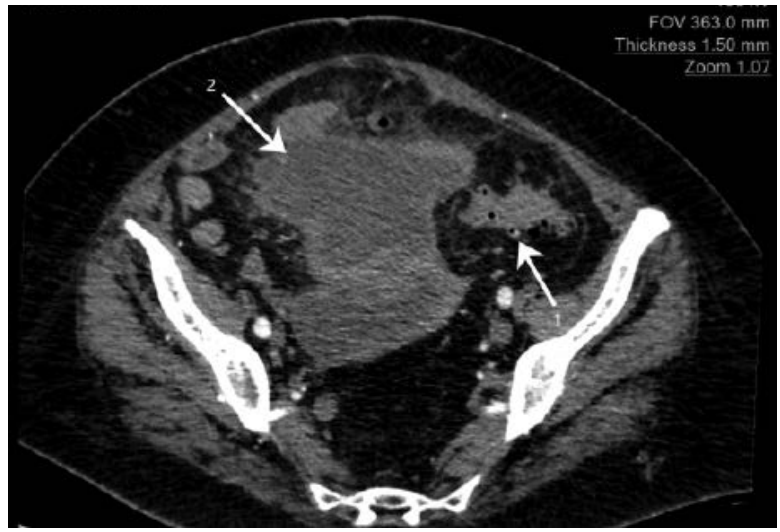

Рисунок 4. Томограмма пациентки А., 62 года. Аксиальный срез. Дивертикулярная болезнь сигмовидной кишки, осложненная формированием абсцесса малого таза. Дивертикул сигмовидной кишки (стрелка 1). Большой абсцесс полости малого таза 121×95×60 мм (стрелка 2).

Figure 4. CT of patient $A$., 62 years old. Diverticular disease of the sigmoid colon, pelvic abscess. Diverticulum of the sigmoid colon (arrow 1). Large abscess of the pelvis $121 \times 95 \times 60 \mathrm{~mm}$ (arrow 2).

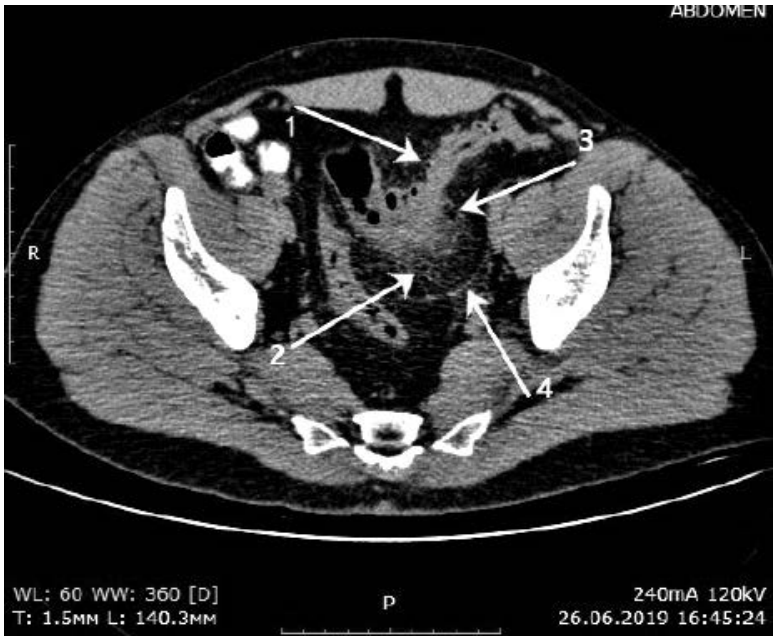

Рисунок 3. Томограмма пациента В., 64 лет. Аксиальный срез. Дивертикулярная болезнь сигмовидной кишки, осложненная дивертикулитом с деструкцией дивертикула. Множественные дивертикулы сигмовидной кишки (стрелка 1). Тяжистость параколической клетчатки (стрелка 2). Пузырек газа вне просвета кишки (стрелка 3). Утолщение корня брыжейки сигмовидной кишки и f. retrocolica (стрелка 4).

Figure 3. CT of patient B., 64 years old. Diverticular disease of the sigmoid colon complicated by diverticulitis. Multiple diverticula of the sigmoid colon (arrow 1). Stranding of paracolic fat (arrow 2). Gas outside the intestinal lumen (arrow 3). Thickening of the mesenteric root of the sigmoid colon and f. retrocolica (arrow 4).

При перфорации дивертикула с формированием абсцесса на РКТ визуализировалась полость с уровнем жидкости и газа в брыжейке или параколической клетчатке, либо в различных отделах брюшной полости. Мы выявили абсцессы у 14 пациентов. При этом у 5 (35,7\%) из них диаметр абсцесса был не более 30 мм, а у 9 (64,3\%)

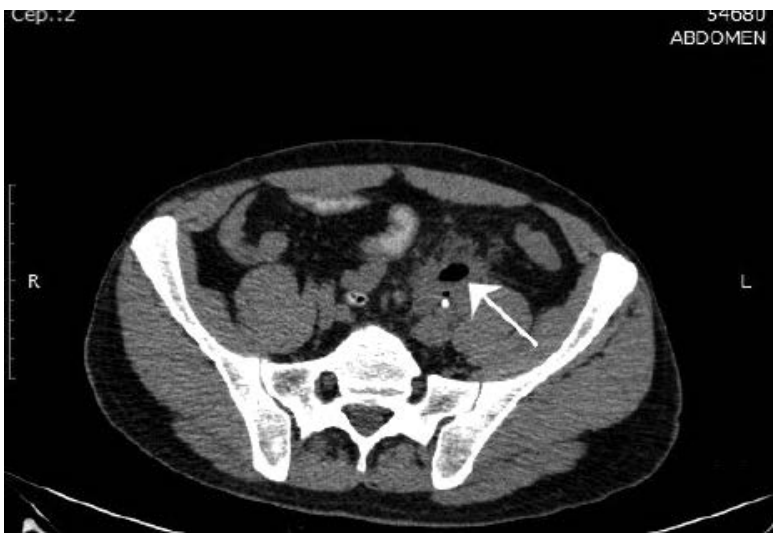

Рисунок 5. Томограмма пациента Х., 41 год. Аксиальный срез. Дивертикулярная болезнь сигмовидной кишки, осложненная формированием параколического абсцесса с горизонтальным уровнем жидкости и газа (стрелка).

Figure 5. CT of patient $X ., 41$ years old. Diverticular disease of the sigmoid colon complicated by pericolic abscess with horizontal fluid level (arrow). 
больных размеры жидкостных образований превышали этот параметр, достигая в одном наблюдении $121 \times 95 \times 60$ мм (Рис. 4-5).

При перитоните дивертикулярного происхождения на КТ отмечали скопления свободной жидкости рядом с пораженным сегментом ободочной кишки, в малом тазу, в различных отделах брюшной полости, а также воспалительную инфильтрацию параколической клетчатки с пузырьками газа, скопление газа в брюшной полости под передней брюшной стенкой (на аксиальных срезах).

При наличии коловезикального свища выявляли прямые (пузырьки газа в просвете мочевого пузыря) и косвенные (утолщение, деформация стенки мочевого пузыря) признаки патологического сообщения между органами (Рис. 6).

В ходе проведенного исследования нами прослежены результаты альтернативного в диагностике острых осложнений ДБОК метода - трансабдоминальной сонографии. Мы применяли трансабдоминальное УзИ в качестве диагностического инструмента первой линии у всех пациентов с подозрением на осложненные формы дивертикулярной болезни. Несомненным достоинством метода являются большая доступность, безопасность и низкая стоимость. Помимо визуализации самих дивертикулов нами отмечены характерные для дивертикулита сонографические признаки: утолщение стенки ободочной кишки и инфильтративные изменения параколической клетчатки. Лишь в 2 из 4 наблюдений ультразвуковое исследование обнаружило признаки перфорации дивертикула - наличие пузырьков воздуха в толще инфильтрированной брыжейки ободочной

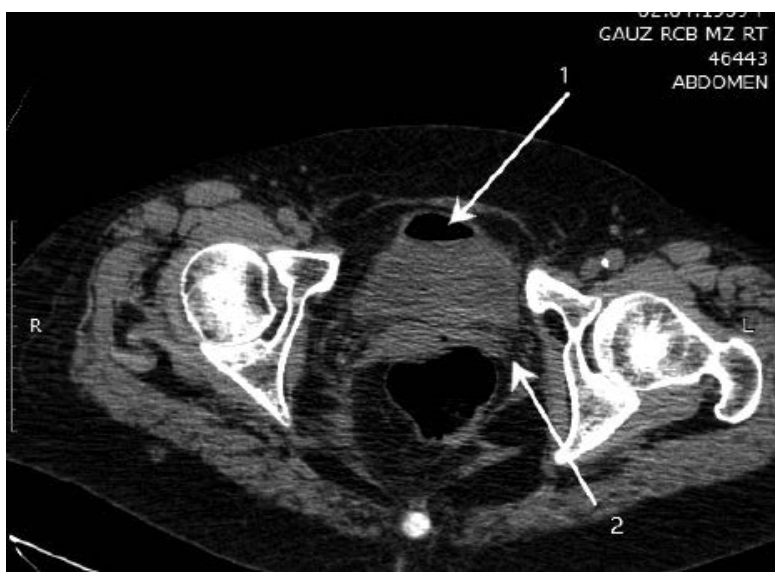

Рисунок 6. Томограмма пациентки В. 58 лет. Аксиальный срез. Дивертикулярная болезнь, осложненная формированием коло-везикального свища. Газ в просвете мочевого пузыря (стрелка 1). Инфильтрация параколической клетчатки (стрелка 2).

Figure 6. CT of patient B. 58 years old. Diverticular disease complicated by colo-vesical fistula (arrow 1). Infiltration of the pericolic fat (arrow 2). кишки. Сравнение этих методов лучевой визуализации в диагностике осложнений ДБОК представляет значительный клинический интерес, однако требует более детального анализа и заслуживает отдельной публикации.

Лечение пациентов с осложненными формами ДБОК проводили в соответствии с национальными клиническими рекомендациями [15]. У всех пациентов с клиникой обострения дивертикулита, острым паракишечным инфильтратом и периколическим абсцессом малого размера (\$3 cm), выявленными на КТ, начинали консервативное лечение, в основе которого - сочетание месалазина и рифаксимина. Консервативное лечение было эффективным у 65 (98,4\%) пациентов этой группы.

Оперированы 24 (26,9\%) пациента. Показаниями к экстренному оперативному лечению были перфоративный дивертикулит, осложненный формированием абсцесса - 9 (37,5\%) или перитонитом - 6 (25\%), острая кишечная непроходимость - 1 (4,2\%). Показания к плановому оперативному лечению включали: частые рецидивы заболевания - 4 (16,6\%), безуспешность консервативной терапии - 1 (4,2\%), стриктура ободочной кишки, осложненная хронической обтурационной толстокишечной непроходимостью - 2 (8,3\%), коловезикальный свищ - 1 (4,2\%).

Малоинвазивные оперативные вмешательства дренирование абсцесса под У3-навигацией выполнены у $4(16,7 \%)$ пациентов. В хирургическом лечении остальных 20 (83,3\%) пациентов с осложнениями ДБОК применен лапаротомный доступ. При этом резекция сигмовидной кишки выполнена у 14 (58,3\%), левосторонняя гемиколэктомия - у 3 (12,5\%) пациентов. У 2 (8,3\%) пациентов с перфоративным дивертикулитом, осложненным формированием абсцесса дугласова пространства, прибегли к комбинированному оперативному вмешательству резекции сигмовидной кишки в сочетании с внутрибрюшинной передней резекцией прямой кишки из-за вовлечения её стенки в формирование капсулы гнойника. В одном (4,2\%) наблюдении у пациента с воспалением дивертикула слепой кишки, осложненного тифлитом, острым гнойным воспалением параколической клетчатки выполнена правосторонняя гемиколэктомия. В 10 (50\%) наблюдениях редукция толстой кишки завершена формированием колоректального анастомоза, выполненного с использованием инвагинационной методики - 7 пациентов, либо с использованием традиционного способа наложения анастомоза с помощью двухрядного кишечного шва - 3 пациента. Двухэтапно, с последующим реконструктивным вмешательством в нашей клинике оперированы 10 (41,6\%) пациентов. Реконструктивные вмешательства выполнялись, 
в среднем, через 3-4 месяца после купирования воспалительного процесса с формированием межкишечного анастомоза двухрядным кишечным швом $6(60,0 \%)$ пациентов, а также инвагинационным способом - $4(40,0 \%)$.

Летальных исходов после оперативных вмешательств не было. Осложнения в послеоперационном периоде возникли у 4 (16,7\%) пациентов: гематома брюшной полости (1), серома послеоперационной раны (1), флебит кубитальной вены (1), острая язва тощей кишки (1). В последнем случае понадобилось выполнение релапаротомии.

у $5(21,3 \%)$ пациентов с инфильтратами дивертикулярного происхождения в течение года возник рецидив заболевания, в связи с чем 3 пациента госпитализированы вновь, повторно проведено консервативное лечение. В 2 оставшихся наблюдениях в связи с формированием периколических абсцессов с признаками местного перитонита по неотложным показаниям выполнено хирургическое вмешательство по типу операции Гартмана. Необходимо отметить, что при первичном поступлении этих пациентов, по данным РКТ, выявляли признаки деструкции (микроперфорации) дивертикула. На КТ такие же признаки отмечены у 2 (8,3\%) пациентов, поступивших повторно и эффективно пролеченных консервативно. Однако один из них через 3 месяца госпитализирован в третий раз по поводу рецидива дивертикулита, осложнившегося перфорацией дивертикула и генерализованным перитонитом, что потребовало экстренного оперативного вмешательства. Данные наблюдения подтверждают мнение о том, что деструкцию дивертикула можно рассматривать в качестве предиктора безуспешности консервативного лечения [4].

Эффективно проведенное дренирование абсцесса под У3-навигацией также не является аргументом в пользу отказа от оперативного вмешательства в период стабилизации состояния пациента, что подтверждают рецидивы этого осложнения. Из 4 пациентов один госпитализирован повторно, произведено повторное дренирование абсцесса по УЗ-навигацией, от предложенного оперативного вмешательства пациент категорически отказался. В другом наблюдении пациент прооперирован через 2 месяца в связи с перфорацией дивертикула, развитием перитонита. Выполнена резекция сигмовидной кишки, наложение десцендостомы с последующей реконструктивновосстановительной операцией. Амбулаторное наблюдение за двумя оставшимися пациентами позволяет констатировать переход воспалительного процесса в хронический с сохранением жалоб на абдоминальный болевой синдром, субфебрильную температуру, а также повышенный уровень лабораторных маркеров воспаления.

\section{ЗАКЛЮЧЕНИЕ}

Компьютерная томография - эффективный метод диагностики при осложненном течении дивертикулярной болезни ободочной кишки, позволяющий своевременно установить клинический вариант воспалительного осложнения, определить показания к оперативному лечению и прогнозировать вероятность рецидива.

\section{УЧАСТИЕ АВТОРОВ:}

Концепция и дизайн исследования: Карпухин 0.Ю., Юсупова А.Ф.

Сбор и обработка материала: Панкратова Ю.С., Черкашина М.И., Ахмадуллина А.А.

Статистическая обработка: Панкратова Ю.С.

Написание текста: Карпухин 0.Ю., Панкратова Ю.С., Черкашина М.И.

Редактирование: Карпухин 0.Ю., Юсупова А.Ф.

\section{AUTHORS CONTRIBUTTON:}

Concept and design of the study: Oleg Yu. Karpukhin, Alsu F. Yusupova

Collection and processing of the material: Yulia S. Pankratova, Malika I. Cherkashina, Albina A. Akhmadullina

Statistical processing: Yulia S. Pankratova

Writing of the text: Oleg Yu. Karpukhin, Yulia S. Pankratova, Malika I. Cherkashina

Editing: Oleg Yu. Karpukhin, Alsu F. Yusupova

\section{СВЕДЕНИЯ ОБ АВТОРАХ}

Карпухин Олег Юрьевич - д.м.Н., профессор кафедры хирургических болезней ФГБОУ В0 Казанский ГМУ Минздрава России, главный внештатный колопроктолог Минздрава РТ

Юсупова Алсу Фаритовна - к.м.н., доцент кафедры онкологии, лучевой диагностики и лучевой терапии ФГБОУ ВО Казанский ГМУ Минздрава России

Панкратова Юлия Сергеевна - аспирант кафедры хирургических болезней ФГБОУ ВО Казанский ГМУ Минздрава России

Черкашина Малика Ильдаровна - врач отделения рентгеновской компьютерной томографии ГАУЗ «Республиканская клиническая больница» МЗ РТ

Ахмадуллина Альбина Айратовна - студентка педиатрического факультета ФГБОУ ВО Казанский ГМУ Минздрава России

\section{ORCID}

Карпухин 0.Ю. - 0000-0002-7479-4945

Юсупова А.Ф. - 0000-0003-2852-3471

Панкратова Ю.С. - 0000-0001-7498-2412

Черкашина М.И. - 0000-0002-7871-042X 
Ахмадуллина А.А. - 0000-0002-7665-1534

INFORMATION ABOUT THE AUTORS (ORCID)

Oleg Yu. Karpukhin -0000-0002-7479-4945

\section{ЛИТЕРАТУРА}

1. Тимербулатов М.В., Куляпин А.В., Лопатин Д.В. и соавт. Диагностическая тактика при ведении больных с дивертикулярной болезнью, осложненной перфоративным дивертикулитом, за 15 лет. Колопроктология. 2018;2:85-88. DOI: 10.33878/2073-75562018-0-2-85-88

2. Minordi LM, Larosa L, Berte $G$, et al. CT of the acute colonic diverticulitis: a pictorial essay. Diagnostic and interventional radiology. 2020;26(6):546-551. D0I: 10.5152/dir.2020.19645

3. Sartelli M, Moore FA, Ansaloni L, et al. A proposal for a CT driven classification of left colon acute diverticulitis. World journal of emergency surgery. 2015; 10, 3. DOI: 10.1186/1749-7922-10-3

4. Poletti PA, Platon A, Rutschmann 0, et al. Acute left colonic diverticulitis: can CT findings be used to predict recurrence? American Journal of Roentgenology. 2004;182(5):1159-65. DOI: 10.2214/ ajr.182.5.1821159. PMID: 1510011.

5. Destigter KK, \& Keating DP. Imaging update: acute colonic diverticulitis. Clinics in colon and rectal surgery. 2009;22(3):147-155. DOI: $10.1055 / \mathrm{s}-0029-1236158$

6. Корнева Е.П., Ростовцев М.В., Нуднов Н.В. и соавт. Возможности КТ в диагностике осложнений дивертикулеза кишечника (клинические наблюдения). Медицинская визуализация. 2019;3:54-65. DOI: 10.24835/1607-0763-2019-3-54-65

7. Andeweg CS, Knobben L, Hendriks JC, et al. How to diagnose acute left-sided colonic diverticulitis: proposal for a clinical scoring system. Annals of surgery. 2011;253(5):940-946. DOI: 10.1097/ SLA.0b013e3182113614

8. Karidis NP, Dimitroulis D, Kouraklis G. The role of abdominal imaging in cases with a high probability of acute left-sided colonic

\section{REFERENCES}

1. Timerbulatov M.V., Kulyapin A.V., Lopatin D.V. et al. Diagnostic tactics in the management of patients with diverticular disease complicated by perforated diverticulitis in 15 years. Koloproktologia. 2018;2:85-88. (In Russ.). DOI: 10.33878/2073-7556-2018-0-2-85-88 2. Minordi LM, Larosa L, Berte G, et al. CT of the acute colonic diverticulitis: a pictorial essay. Diagnostic and interventional radiology. 2020;26(6):546-551. DOI: 10.5152/dir.2020.19645

3. Sartelli M, Moore FA, Ansaloni L, et al. A proposal for a CT driven classification of left colon acute diverticulitis. World journal of emergency surgery. 2015; 10, 3. D0I: 10.1186/1749-7922-10-3

4. Poletti PA, Platon A, Rutschmann 0, et al. Acute left colonic diverticulitis: can CT findings be used to predict recurrence? American Journal of Roentgenology. 2004;182(5):1159-65. DOI: 10.2214/ ajr.182.5.1821159. PMID: 1510011.

5. Destigter KK, \& Keating DP. Imaging update: acute colonic diverticulitis. Clinics in colon and rectal surgery. 2009;22(3):147-155. DOI: $10.1055 / \mathrm{s}-0029-1236158$

6. Korneva E.P., Rostovtsev M.V., Nudnov N.V. et al. Possibilities of $\mathrm{CT}$ in the diagnosis of complications of intestinal diverticulosis (clinical observation). Medical Visualization. 2019;3:54-65. (In Russ.). DOI: 10.24835/1607-0763-2019-3-54-65

7. Andeweg CS, Knobben L, Hendriks JC, et al. How to diagnose acute left-sided colonic diverticulitis: proposal for a clinical scoring system. Annals of surgery. 2011;253(5):940-946. D0I: 10.1097/ SLA.0b013e3182113614.

8. Karidis NP, Dimitroulis D, Kouraklis G. The role of abdominal
Alsu F. Yusupova - 0000-0003-2852-3471

Yulia S. Pankratova - 0000-0001-7498-2412

Malika I. Cherkashina - 0000-0002-7871-042X

Albina A. Akhmadullina - 0000-0002-7665-1534

diverticulitis based on a clinical scoring system. Annals of surgery.2013;258(2):1-4. D0I: 10.1097/SLA.0b013e31829c7a9d

9. Toorenvliet BR, Bakker RFR., Breslau PJ, et al. Colonic diverticulitis: a prospective analysis of diagnostic accuracy and clinical decision-making. Colorectal Disease. 2010;12(3):179-186. DOI: $10.1111 / j .1463-1318.2009 .01778 . x$

10. Kaiser AM, Jiang JK, Lake JP, et al. The management of complicated diverticulitis and the role of computed tomography. American Journal of Gastroenterology. 2005;100(4):910-917. DOI: $10.1111 / j .1572-0241.2005 .41154 . x$

11. Baker M.E. Imaging and interventional techniques in acute left sided diverticulitis. Journal of Gastrointestinal Surgery. 2008;12(8):1314-1317. D0I: 10.1007/s11605-008-0490-2

12. Белов Д.М., Зароднюк И.В., Михальченко В.А. Компьютернотомографическая диагностика воспалительных осложнений дивертикулярной болезни ободочной кишки (обзор литературы). Колопроктология. 2016; 4:. 60-68

13. Kandagatla PG, Stefanou AJ. Current Status of the Radiologic Assessment of Diverticular Disease. Clinics in colon and rectal surgery. 2018;31(4):217-220. DOI: 10.1055/s-0037-1607466

14. Pereira JM, Sirlin CB, Pinto PS, et al. Disproportionate fat stranding: a helpful CT sign in patients with acute abdominal pain. Radiographics. 2004;24(3):703-15. DOI: 10.1148/rg.243035084

15. Ардатская М.Д., Ачкасов С.И., Веселов В.В., и соавт. Клинические рекомендации. Дивертикулярная болезнь. Колопроктология. 2021;20(3):10-27. DOI: 10.33878/2073-75562021-20-3-10-27

imaging in cases with a high probability of acute left-sided colonic diverticulitis based on a clinical scoring system. Annals of surgery. 2013;258(2):1-4. DOI: 10.1097/SLA.0b013e31829c7a9d

9. Toorenvliet BR, Bakker RFR., Breslau PJ, et al. Colonic diverticulitis: a prospective analysis of diagnostic accuracy and clinical decision-making. Colorectal Disease. 2010;12(3):179-186. DOI: $10.1111 / \mathrm{j} .1463-1318.2009 .01778 . x$

10. Kaiser AM, Jiang JK, Lake JP, et al. The management of complicated diverticulitis and the role of computed tomography. American Journal of Gastroenterology. 2005;100(4):910-917. DOI: $10.1111 / j .1572-0241.2005 .41154 . x$

11. Baker M.E. Imaging and interventional techniques in acute left sided diverticulitis. Journal of Gastrointestinal Surgery. 2008;12(8):1314-1317. D0I: 10.1007/s11605-008-0490-2

12. Belov D.M., Zarodnyuk I.V., Mikhalchenko V.A.etal. Computed tomography diagnostic of inflammatory complications of colon diverticulitis (review). Koloproktologia. 2016;4:60-68. (In Russ.).

13. Kandagatla PG, Stefanou AJ. Current Status of the Radiologic Assessment of Diverticular Disease. Clinics in colon and rectal surgery. 2018;31(4):217-220. DOI: 10.1055/s-0037-1607466

14. Pereira JM, Sirlin CB, Pinto PS, et al. Disproportionate fat stranding: a helpful CT sign in patients with acute abdominal pain. Radiographics. 2004;24(3):703-15. D0I: 10.1148/rg.243035084 15. Ardatskaya M.D., Achkasov S.I., Veselov V.V. et al. Clinical guidelines. Diverticular desease. Koloproktologia. 2021;20(3):10-27. D0I: 10.33878/2073-7556-2021-20-3-10-27 